\title{
Psychiatric traits in patients with vasovagal and unexplained syncope
}

This article was published in the following Dove Press journal: International Journal of General Medicine

\author{
Abdullah Alhuzaimi' \\ Alwaleed Aljohar ${ }^{2}$ \\ Ahmad N. Alhadi ${ }^{3,4}$ \\ Abdulqudous Aljenedil ${ }^{2}$ \\ Ahmad S Hersi' \\ 'Department of Cardiac Sciences, \\ King Fahad Cardiac Center, College \\ of Medicine, King Saud University, \\ Riyadh, Kingdom of Saudi Arabia; \\ ${ }^{2}$ College of Medicine, King Saud \\ University, Riyadh, Kingdom of Saudi \\ Arabia; ${ }^{3}$ Department of Psychiatry, \\ College of Medicine, King Saud \\ University, Riyadh, Kingdom of Saudi \\ Arabia; ${ }^{4}$ SABIC Psychological Health \\ Research \& Applications Chair \\ (SPHRAC), College of Medicine, King \\ Saud University, Riyadh, Kingdom of \\ Saudi Arabia
}

Purpose: Syncope is a common condition affecting almost one-third of the general population. The present study measures the prevalence of psychiatric traits in patients presenting with syncope (unexplained and vasovagal) and whether recurrent attacks have an impact on psychiatric profiles. Patients and methods: This is a case-control study in a tertiary hospital enrolling all patients aged $\geq 12$ years with single or recurrent syncopal attacks. A self-reporting psychometric questionnaire (The Symptoms Checklist-90-Revised) was used to screen for depression, anxiety, somatization disorder, and phobia. Crude comparisons of average scores were done. Further, multiple logistic regression analyses were carried out to measure the impact of syncope on each psychiatric domain. The control group were matched for age, gender, and chronic illnesses with a ratio of $1: 3$

Results: There were 43 cases and 129 control subjects, with predominance of females $(67.4 \%)$ and an average age of 33.8 years (standard deviation $=16$ ). There were no significant differences in average scores of depression (13 vs $14.53, P=0.31)$, anxiety $(11.3$ vs $10.4, P=0.51)$, or phobia (5.4 vs 5.2, $P=0.88$ ). However, the syncope group had a higher average score for somatization disorder (18.53 vs $13.66, P=0.002)$. Binary logistic regression model showed that the association between syncope and somatization disorder was independent of competing confounders (odds ratio $=3.75,95 \%$ confidence interval: $1.72,8.15, P=0.001$ ). A sub-analysis of the case group showed that patients with multiple syncopal attacks (six or more) had higher average scores of depression, anxiety, phobia, and somatization disorder compared to those who had less than six attacks.

Conclusion: Syncope was independently associated with somatization disorder traits. Further, recurrent syncope resulted in greater deterioration of patients' psychiatric profiles. Thus, taking into account the psychiatric status in the management of such patients is crucial.

Keywords: syncope, psychiatric disorders, anxiety, somatization disorder, phobia, depression

\section{Introduction}

Syncope is defined as transient loss of consciousness (LOC) resulting from global cerebral hypoperfusion characterized by loss of postural tone followed by spontaneous recovery. It is majorly classified into cardiogenic, neurally mediated (ie, vasovagal syncope [VVS]), or orthostatic in etiology. ${ }^{1,2}$ The lifetime risk of experiencing syncope is difficult to estimate owing to the variance according to age and gender. ${ }^{1}$ However, in one study, the incidence of syncope in patients aged between 35 and 60 reached $35 \%{ }^{3}$ Syncope was estimated to account for roughly $1 \%$ of all emergency department visits. ${ }^{4}$ Syncope is not only a prevalent and morbid symptom but also challenging to
Correspondence: Abdullah Alhuzaimi Department of Cardiac Sciences, King Fahad Cardiac Center, College of Medicine, King Saud University, PO Box 7805, Riyadh II472, Kingdom of Saudi Arabia

Tel +966 || $467 \mid 609$

Fax +966 II 467 I232

Email Aalhuzaimi@ksu.edu.sa 
diagnose. Despite the emergence of Head-Up Table Tilt test (HUT) and its utilization in diagnosing VVS, a great number of cases remain undiagnosed, ${ }^{5,6}$ where it is said to be unexplained syncope (US).

Syncope is a limiting disease which may force patients to undergo major lifestyle modification such as employment adjustments, friends and spouses conflicts, avoidance of driving, ${ }^{7,8}$ and major injuries secondary to falls. ${ }^{8-11}$ In addition, it was associated with impaired quality of life..$^{9,12-14}$ Therefore, the psychological and psychiatric profiles may be altered in such population. This hypothesis has been tested before with discrepant results. ${ }^{7-11,14-17}$ Among all psychiatric disorders that were screened for, anxiety was the most commonly reported,,$^{9,11,14,16,17}$ followed by depression. ${ }^{7,9,17}$ Somatization and phobic disorders were not as thoroughly investigated, but they were found to be associated with syncope in some reports. ${ }^{7,9,14}$

Psychiatric symptoms at baseline predicted higher rates of recurrence during follow-up. ${ }^{9,15,16,18}$ Additionally, two studies have shown that psychiatric interventions had a positive effect in syncope outcomes and response to conventional therapy. ${ }^{17,19}$ In the present study, we aimed to measure the prevalence of depression, anxiety, somatization disorder, and phobia in patients suffering from VVS or US. Further, we aimed to explore the effect of recurrent syncope on patients' psychiatric profile and whether it was associated with worse psychiatric outcomes.

\section{Patients and methods Syncope definition}

The diagnosis of syncope was based on the latest guidelines by the European Society of Cardiology. ${ }^{1}$ Patients having nearfaints or loss of postural tone without complete LOC were not included. To identify the patients, a generated search for the word "syncope" or its equivalents was carried out in all electronic medical charts and reports of our echocardiography and electrophysiology laboratories and clinics. Afterward, a careful investigation of medical documentation, laboratory investigations, medications, electrocardiogram, and echocardiography and HUT reports was performed. Medical histories regarding frequency of attacks, family history of syncope, and chronic illnesses such as hypertension and diabetes mellitus were obtained.

\section{Participants}

Patients were eligible for the study if they had experienced at least one attack of complete LOC and sought medical care under cardiac services between August 2009 and August
2014 at King Fahad Cardiac Center, Riyadh, Saudi Arabia. Exclusion criteria were syncope resulting from major cardiovascular diseases (valvular heart disease, structural heart disease, or cardiac arrhythmias), a previous diagnosis of mental disorders or being actively on psychiatric therapy, or LOC secondary to a neurological etiology. Initially, 112 patients were enrolled for screening. Eighteen individuals were excluded due to major cardiac and structural heart diseases. In addition, 41 patients were not able to be contacted and 10 patients refused to participate. Therefore, the final number of cases was 43 patients. A control group from the general population was recruited matching for age, gender, and chronic illnesses. The control group recruitment was done via spreading the examination tool for self-reporting and then random selection was done for the final analysis. To assure that the control group did not experience syncopal attacks throughout their life, a question to this regard was added in their questionnaire and their participation was eliminated if they had experienced syncope. With a case:control ratio of $1: 3$, the final sample size was formed by 167 individuals. An informed verbal consent prior to participation was obtained from both groups. For participats under the age of 18, their parents or legal guardians provided the consent on their behalf. The study protocol and consent process was approved by the Institutional Review Board of King Khalid University Hospital, King Saud University, Riyadh, Saudi Arabia.

\section{Psychiatric evaluation}

All patients completed selected sections of the SymptomsChecklist-90-Revised (SCL-90-R) scale in its Arabic version via a telephone interview. The SCL-90-R is a current point-of-time measurement scale that is being widely used to screen for nine psychiatric disorders and was designed for appropriate use among the general public and medically ill patients. ${ }^{20}$ It is composed of 90 items, where each item measures the severity of a given psychiatric symptom by a score ranging from 0 to 4 . Subsequently, a total score for each entity is calculated, and a high score indicates greater severity. Our subjects completed four domains which include 44 items that screened for depression, anxiety, somatization disorder, and phobia.

\section{Statistical analysis}

Categorical data were summarized with absolute numbers and percentages. Numeric data were summarized using means and standard deviation (SD). Comparison between the two groups was performed using chi-square test for categorical variables and independent sample $t$-test for continuous 
variables. In order to assess the impact of recurrent syncope, we divided the case group according to the frequency of attacks (below six attacks vs six attacks or more) and compared the average scores of each psychiatric entity between them. Adjusted odds ratios (ORs) were carried out using multiple logistic regression after grouping the cohort into above and below the median value. Adjustments were made for age, gender, and chronic illnesses. Statically significant results were attained whenever the $P$-value of the statistical test was below 0.05 . Statistical analyses were performed via SPSS 21.0 (IBM Corporation, Armonk, NY, USA).

\section{Results}

\section{Baseline characteristics}

Ninety-four patients initially met the inclusion criteria for further interviewing, of whom, 43 completed the study questionnaire. The average age was $34.7 \pm 18$ years (range 14-72). Twenty-nine patients (67.4\%) were females and 17 $(39.5 \%)$ had a history of chronic illnesses. Almost half the patients $(53.5 \%)$ experienced less than six syncopal attacks (median $=2)$. HUT was performed in $27(62.8 \%)$ patients and was positive in $19(44.2 \%)$. All baseline demographics of the cases were of no difference compared to controls (Table 1).

\section{Prevalence of psychiatric illnesses}

Comparison between patients and controls revealed no significant difference in the average scores of depression, anxiety, or phobia. However, somatization disorder was significantly higher among patients than among controls (18.5 vs $13.7 \%, P=0.002)$. All comparisons are presented in Table 2 .

Two groups were identified according to median scores (above and below median), where median scores for depression, anxiety, somatization disorder, and phobia were 12.5 ,

Table I Baseline characteristics of cases compared to controls

\begin{tabular}{lll}
\hline & Cases (N = 43) & Controls (N = I 24) \\
\hline Demographics & & \\
Age (years), mean (SD) & $34.7(18.5)$ & $33.8(16.1)$ \\
Female, $\mathrm{n}(\%)$ & $29(67.4)$ & $87(67.4)$ \\
Chronic illnesses, $\mathrm{n}(\%)$ & $17(39.5)$ & $50(38.8)$ \\
Frequency of attacks & & \\
Below six attacks, $\mathrm{n}(\%)$ & $23(53.5)$ & - \\
Six attacks or more, $\mathrm{n}(\%)$ & $20(46.5)$ & - \\
HUT results & & - \\
Positive, $\mathrm{n}(\%)$ & $19(44.2)$ & - \\
Negative, $\mathrm{n}(\%)$ & $8(18.6)$ & - \\
Not performed, $\mathrm{n}(\%)$ & $16(37.2)$ & \\
\hline
\end{tabular}

Abbreviations: SD, standard deviation; HUT, Head-Up Table Tilt test.
Table 2 Psychometric average scores for cases compared to controls

\begin{tabular}{|c|c|c|c|}
\hline & $\begin{array}{l}\text { Cases } \\
(N=43)\end{array}$ & $\begin{array}{l}\text { Controls } \\
(N=124)\end{array}$ & $P$-value \\
\hline Depression, mean (SD) & $13.0(8.3)$ & $14.5(8.4)$ & 0.310 \\
\hline Anxiety, mean (SD) & $11.3(7.8)$ & $10.4(7.1)$ & 0.515 \\
\hline Somatization disorder, mean (SD) & $18.5(8.6)$ & $13.7(8.8)$ & 0.002 \\
\hline Phobia, mean (SD) & $5.4(5.7)$ & $5.2(4.7)$ & 0.889 \\
\hline
\end{tabular}

Abbreviation: SD, standard deviation

Table 3 Crude and adjusted odds ratios (ORs) obtained using multiple logistic regression to assess the association between syncope and scores above median values for psychiatric traits for cases compared to controls

\begin{tabular}{lllll}
\hline Outcomes & $\begin{array}{l}\text { Crude OR } \\
(\mathbf{9 5 \%} \mathbf{~ C I})\end{array}$ & P-value & $\begin{array}{l}\text { Adjusted OR } \\
(\mathbf{9 5 \%} \mathbf{~ C l})\end{array}$ & P-value \\
\hline Depression & $0.8(0.42,1.66)$ & 0.598 & $0.8(0.40,1.68)$ & 0.598 \\
Anxiety & $1.5(0.73,2.90)$ & 0.292 & $1.5(0.74,3.14)$ & 0.255 \\
$\begin{array}{l}\text { Somatization } \\
\text { disorder }\end{array}$ & $3.5(1.64,7.37)$ & 0.001 & $3.8(1.72,8.15)$ & 0.001 \\
Phobia & $0.8(0.41,1.66)$ & 0.596 & $0.8(0.41,1.70)$ & 0.618 \\
\hline
\end{tabular}

Note: Adjusted for age, gender, and chronic illnesses.

Abbreviation: $\mathrm{Cl}$, confidence interval.

9, 13.5, and 4, respectively. After adjusting for age, gender, and chronic illnesses, there remained a significant association between syncope and somatization disorder symptoms $(\mathrm{OR}=3.75,95 \%$ confidence interval $[95 \% \mathrm{CI}]: 1.72,8.15$, $P=0.001)$. Table 3 shows the crude and adjusted ORs for all four domains.

As the somatization disorder section in the SCL-90-R contains a question on whether an individual had experienced previous "near fainting" attacks or not, this may result in inaccurate and biased estimations. Therefore, we performed a sensitivity analysis by removing this question and reanalyzing the data, and the findings remained unaltered in both crude $(\mathrm{OR}=2.79,95 \% \mathrm{CI}: 1.35,5.77, P=0.006)$ and adjusted $(\mathrm{OR}$ $=2.94,95 \% \mathrm{CI}: 1.4,6.2, P=0.005)$ analyses.

\section{Recurrent syncope}

A sub-analysis of the case group was carried out $(\mathrm{N}=43)$. Patients were divided into two groups according to the frequency of syncopal attacks: below six attacks $(\mathrm{N}=23)$ vs six attacks or more $(\mathrm{N}=20)$. As shown in Table 4 , average scores of depression, anxiety, and somatization disorder were significantly higher among those experiencing six attacks or more $(16.3$ vs $10.2 \%, P=0.018 ; 14.3$ vs $8.7 \% ; P=0.018$, 21.5 vs $16 \%, P=0.037$, respectively). In addition, the average score of phobia was higher in the case group, but with marginal statistical significance ( 7.2 vs $3.8 \%, P=0.055)$. 
Table 4 Psychometric average scores comparisons of the case group based on recurrence of syncope

\begin{tabular}{|c|c|c|c|}
\hline & $\begin{array}{l}\text { Below six } \\
(N=23)\end{array}$ & $\begin{array}{l}\text { Six or more } \\
(N=20)\end{array}$ & $P$-value \\
\hline Depression, mean (SD) & $10.2(5.9)$ & $16.3(9.5)$ & 0.018 \\
\hline Anxiety, mean (SD) & $8.7(6.0)$ & |4.3 (8.8) & 0.018 \\
\hline $\begin{array}{l}\text { Somatization disorder, } \\
\text { mean (SD) }\end{array}$ & $16.0(8.1)$ & $21.5(8.4)$ & 0.037 \\
\hline Phobia, mean (SD) & $3.8(4.4)$ & $7.1(6.7)$ & 0.055 \\
\hline
\end{tabular}

Abbreviation: SD, standard deviation.

Table 5 Crude and adjusted odds ratios (ORs) obtained using multiple logistic regression to assess the association between psychiatric traits and the frequency of syncopal attacks (less than six vs six or more)

\begin{tabular}{lllll}
\hline Outcomes & $\begin{array}{l}\text { Crude OR } \\
(\mathbf{9 5 \%} \mathbf{~ C l})\end{array}$ & P-value & $\begin{array}{l}\text { Adjusted OR } \\
(\mathbf{9 5 \%} \mathbf{~ C l})\end{array}$ & $P$-value \\
\hline Depression & $2.3(0.69,7.95)$ & 0.175 & $2.5(0.70,9.10)$ & 0.159 \\
Anxiety & $3.5(0.99,12.24)$ & 0.052 & $3.7(0.95,13.96)$ & 0.059 \\
$\begin{array}{l}\text { Somatization } \\
\text { disorder }\end{array}$ & $5.3(1.45,19.67)$ & 0.012 & $5.4(1.31,21.95)$ & 0.020 \\
Phobia & $2.8(0.80,9.76)$ & 0.107 & $2.8(0.76,10.19)$ & 0.125 \\
\hline
\end{tabular}

Note: Adjusted for age, gender, and chronic illnesses.

Abbreviation: $\mathrm{Cl}$, confidence interval.

In addition, patients with syncope were grouped according to the median score, and after adjusting for confounders, all four domains had a strong association with recurrent syncope, although statistical significance was observed with somatization disorder only $(\mathrm{OR}=5.36,95 \% \mathrm{CI}$ : $1.31,21.95$, $P=0.020)$ (Table 5).

\section{Discussion}

\section{Prevalence of psychiatric symptoms}

In this observational study, we could not show an overwhelming prevalence of psychiatric symptoms in patients with syncope, except, however, in somatization disorder. Several reports have established an association between multiple psychiatric disorders and syncope, ${ }^{7-11,14-17}$ with depression and anxiety being the most commonly reported. Relatively fewer reports investigated the prevalence of somatization disorder in syncope patients and reported discrepant results. Somatization disorder was found to be prevalent in a singlearmed study by Linzer et al. ${ }^{7}$ This was confirmed by another report after recruiting a control group. ${ }^{9}$ These findings are in concordance with our data. Nonetheless, two other studies did not show a significant relationship. ${ }^{14,17}$

The divergence in results may be explained by several reasons. First, some studies have included patients experiencing presyncope without complete LOC, $7,9,14$ which may be less burdensome on patients, and others confined their recruitment to more than two episodes of complete LOC. ${ }^{8,15-17}$ Second, different measurement scales for psychiatric symptoms were included..$^{8-11,14-16}$ Finally, not all studies were matched with a control group. . $^{7,10,14,15,17}$

Indeed, somatization disorder can only be diagnosed if medical diseases were excluded. The high prevalence of somatization disorder symptoms we found can be explained by the overlap between the prodromal symptoms of VVS and somatization disorder. Nevertheless, this does not trivialize the coexistence of somatization disorder with syncope, and further exploration of their relationship is essential.

\section{Recurrent syncope}

The highlight of our data is the significant deterioration of psychosomatic status associated with recurrent syncope. Patients experiencing six attacks or more were at higher risk of having psychiatric symptoms. This is in agreement with a study by Lee et al in regards to anxiety, but not depression, ${ }^{10}$ and another report showed no significant relationship between the frequency of syncopal attacks and psychiatric disorders. ${ }^{9}$

Whether psychiatric distress is a cause or a result of recurrent syncope remains unclear. Recurrent syncope can be distressing, and its negative impact may manifest as a psychosocial impairment. This can be due to its major limitation of daily life activities, where patients experienced adjustment of employment, ${ }^{7}$ conflicts with spouses and friends, ${ }^{7}$ major injuries secondary to falls, ${ }^{8-11}$ and using avoidant coping strategies making them dependent on others. ${ }^{8,13,16}$ In addition, when patients were asked about their psychiatric symptoms, the majority attributed them to experiencing syncopal attacks rather than the opposite. ${ }^{7,12}$ Finally, the fact that psychosocial impairment was observed in patients with other chronic diseases such as chronic obstructive lung disease, ${ }^{21}$ end-stage renal disease, ${ }^{22}$ and heart failure ${ }^{23,24}$ suggests that syncope may be a trigger for experiencing psychiatric traits.

On the other hand, psychiatric disorders at the time of diagnosis predicted recurrence of syncope during followup, $, 15,16,18$ and when patients underwent psychiatric interventions, syncopal attacks became less frequent or completely resolved as opposed to their recurrence among those refusing psychiatric therapy. ${ }^{17,19}$ Furthermore, selective serotonin reuptake inhibitors (SSRIs) showed positive outcomes in patients suffering from refractory recurrent syncope, ${ }^{25-27}$ emphasizing the importance of taking into account the psychiatric status in managing patients with syncope, and that syncope may subsequently evolve secondary to psychiatric distress. In support of this theory, the American Psychiatric 
Association included syncope as one of the presenting symptoms of panic, somatization, and phobic disorders in the Diagnostic and Statistical Manual of Mental Disorders, fifth edition, ${ }^{28}$ where functional impairment is required to confirm the diagnosis.

We propose a theory that may partially explain these contradictions. Patients experiencing single or infrequent intermittent attacks that do not interfere with their lives maintain an intact psychiatric well-being. However, if these attacks resume to the extent that functional impairment is evident, patients might go into a vicious cycle of altered illness perception, resulting in deterioration of their psychiatric status, which, in turn, leads to further recurrence of syncopal attacks.

\section{Clinical implications}

Since psychiatric disorders at the time of diagnosis predicted recurrence during follow-up, ${ }^{9,15,16}$ this will encourage practitioners who encounter patients with syncope to address psychiatric issues using simple screening tools such as SCL90-R for subsequent referral to psychiatric or psychological services, if indicated. Not only did cognitive behavioral therapy show a better response to conventional syncope management, ${ }^{17,19}$ but also pharmacological measures such as SSRIs. ${ }^{26,27}$ Unfortunately, however, the refusal rate of screening for psychiatric symptoms was high. Out of all patients who were reachable, nearly $20 \%$ refused to participate. This is in agreement with Ventura et al, ${ }^{17}$ where almost half their patients declined a simple psychiatric interview fearing to receive a diagnosis of a psychiatric disorder, because of public stigma, prejudice, and discrimination toward mentally ill patients, ${ }^{29,30}$ which subsequently leads to under-recognition of major psychiatric disorders. Alternatively, one may postulate that simple reassurance and education about the disease's benign nature and positive prognosis may minimize its negative effect and improve outcomes. Unfortunately, this approach has not shown significant improvement in outcomes when compared to expert interventions. ${ }^{15}$ Therefore, the management of such disease requires aiming for both conventional treatment and psychiatric therapy.

\section{Study limitations}

Our study has a small sample size which may have influenced our findings. Our patients constituted of those referred to cardiac services, hence we may have missed patients experiencing syncope investigated under the care of other services. The number of patients who underwent HUT was small and, therefore, we could not examine whether or not the results of HUT correlated with worse psychiatric profiles. Further, the lack of HUT results rendered us unable to definitely exclude patients presenting with pseudosyncope. The symptoms-checklist (SCL-90-R) utilized in our study remains a screening tool for psychiatric symptoms and cannot be used as a diagnostic scale.

\section{Conclusion}

In conclusion, we found a high prevalence of somatization disorder symptoms among patients having unexplained and vasovagal syncope. Furthermore, recurrent syncope was associated with a greater deterioration of patients' psychiatric profile. This highlights the importance of psychiatric screening among patients experiencing refractory syncopal disease to determine the need for further multidisciplinary management.

\section{Acknowledgments}

This research was financially supported in full by SABIC Psychological Health Research and Applications Chair, Department of Psychiatry, College of Medicine, Deanship of Post Graduate Teaching, King Saud University, Riyadh, Saudi Arabia.

The abstract of this paper was presented at the 27th Saudi Heart Association Symposium on February 2016 in Riyadh, Saudi Arabia, as a conference talk with interim findings. The abstract was published in the Journal of the Saudi Heart Association: https://doi.org/10.1016/j.jsha.2016.04.012.

\section{Disclosure}

The authors report no conflicts of interest in this work.

\section{References}

1. The European Society of Cardiology Guidelines for the diagnosis and management of syncope reviewed by Angel Moya, MD, FESC, Chair of the Guideline Taskforce with J. Taylor, MPhil. Eur Heart J. 2009;30(21):2539-2540.

2. Puppala VK, Dickinson O, Benditt DG. Syncope: classification and risk stratification. J Cardiol. 2014;63(3):171-177.

3. Ganzeboom KS, Mairuhu G, Reitsma JB, Linzer M, Wieling W, van Dijk N. Lifetime cumulative incidence of syncope in the general population: a study of 549 Dutch subjects aged 35-60 years. J Cardiovasc Electrophysiol. 2006;17(11):1172-1176.

4. Probst MA, Kanzaria HK, Gbedemah M, Richardson LD, Sun BC. National trends in resource utilization associated with ED visits for syncope. Am J Emerg Med. 2015;33(8):998-1001.

5. Mohan L, Lavania AK. Vasovagal syncope: an enigma. J Assoc Physicians India. 2004;52:301-304.

6. Tan MP, Duncan GW, Parry SW. Head-up Tilt Table Testing: a state-ofthe-art review. Minerva Med.2009;100(4):329-338.

7. Linzer M, Pontinen M, Gold DT, Divine GW, Felder A, Brooks WB. Impairment of physical and psychosocial function in recurrent syncope. J Clin Epidemiol. 1991;44(10):1037-1043.

8. Shaffer C, Jackson L, Jarecki S. Characteristics, perceived stressors, and coping strategies of patients who experience neurally mediated syncope. Heart Lung. 2001;30(4):244-249. 
9. Giada F, Silvestri I, Rossillo A, Nicotera PG, Manzillo GF, Raviele A. Psychiatric profile, quality of life and risk of syncopal recurrence in patients with tilt-induced vasovagal syncope. Europace. 2005;7(5):465-471.

10. Lee SH, Park SJ, Byeon K, On YK, Yim HR, Kim JS. Prevalence and clinical factors of anxiety and depression in neurally mediated and unexplained syncope. Yonsei Med J. 2013;54(3):583-589.

11. Zysko D, Szewczuk-Boguslawska M, Kaczmarek M, et al. Reflex syncope, anxiety level, and family history of cardiovascular disease in young women: case-control study. Europace. 2015;17(2):309-313.

12. Andrighetto AG, John AB, Barbisan JN, Taborda JG. Medically unexplained syncope and its relationship to psychiatric disorders. Arq Bras Cardiol. 1999;72(6):751-760.

13. Gracie J, Newton JL, Norton M, Baker C, Freeston M. The role of psychological factors in response to treatment in neurocardiogenic (vasovagal) syncope. Europace. 2006;8(8):636-643.

14. Rafanelli C, Gostoli S, Roncuzzi R, Sassone B. Psychological correlates of vasovagal versus medically unexplained syncope. Gen Hosp Psychiatry. 2013;35(3):246-252.

15. D'Antono B, Dupuis G, St-Jean K, et al. Prospective evaluation of psychological distress and psychiatric morbidity in recurrent vasovagal and unexplained syncope. J Psychosom Res. 2009;67(3):213-222.

16. Kouakam C, Lacroix D, Klug D, Baux P, Marquie C, Kacet S. Prevalence and prognostic significance of psychiatric disorders in patients evaluated for recurrent unexplained syncope. Am J Cardiol. 2002;89(5): 530-535.

17. Ventura R, Maas R, Ruppel R, et al. Psychiatric conditions in patients with recurrent unexplained syncope. Europace. 2001;3(4):311-316.

18. Kapoor WN, Fortunato M, Hanusa BH, Schulberg HC. Psychiatric illnesses in patients with syncope. Am J Med. 1995;99(5):505-512.

19. Newton JL, Kenny RA, Baker CR. Cognitive behavioural therapy as a potential treatment for vasovagal/neurocardiogenic syncope - a pilot study. Europace. 2003;5(3):299-301.
20. Derogatis LR, Unger R. Symptom Checklist-90-Revised. The Corsini Encyclopedia of Psychology. USA: John Wiley \& Sons, Inc.; 2010.

21. Chaudhary SC, Nanda S, Tripathi A, et al. Prevalence of psychiatric comorbidities in chronic obstructive pulmonary disease patients. Lung India. 2016;33(2):174-178.

22. Kilicoglu AG, Bahali K, Canpolat N, et al. Impact of end stage renal disease on psychological status and quality of life. Pediatr Int. 2016;58(12):1316-1321.

23. Picco L, Subramaniam M, Abdin E, Vaingankar JA, Chong SA. Prevalence and correlates of heart disease among adults in Singapore. Asian J Psychiatry. 2016;19:37-43.

24. Easton K, Coventry P, Lovell K, Carter LA, Deaton C. Prevalence and measurement of anxiety in samples of patients with heart failure: metaanalysis. J Cardiovasc Nurs. 2016;31(4):367-379.

25. Vyas A, Swaminathan PD, Zimmerman MB, Olshansky B. Are treatments for vasovagal syncope effective? A meta-analysis. Int J Cardiol. 2013;167(5):1906-1911.

26. Grubb BP, Samoil D, Kosinski D, Kip K, Brewster P. Use of sertraline hydrochloride in the treatment of refractory neurocardiogenic syncope in children and adolescents. J Am Coll Cardiol. 1994;24(2):490-494.

27. Di Girolamo E, Di Iorio C, Sabatini P, Leonzio L, Barbone C, Barsotti A. Effects of paroxetine hydrochloride, a selective serotonin reuptake inhibitor, on refractory vasovagal syncope: a randomized, double-blind, placebo-controlled study. J Am Coll Cardiol. 1999;33(5):1227-1230.

28. American Psychiatric Association. Diagnostic and Statistical Manual of Mental Disorders (DSM-5®). Washington, DC: American Psychiatric Association Publishing; 2013.

29. Ye J, Chen TF, Paul D, et al. Stigma and discrimination experienced by people living with severe and persistent mental illness in assertive community treatment settings. Int J Soc Psychiatry. 2016;62(6):532-541.

30. Kao YC, Lien YJ, Chang HA, Wang SC, Tzeng NS, Loh CH. Evidence for the indirect effects of perceived public stigma on psychosocial outcomes: The mediating role of self-stigma. Psychiatry Res. 2016;240:187-195.
International Journal of General Medicine

\section{Publish your work in this journal}

The International Journal of General Medicine is an international, peer-reviewed open-access journal that focuses on general and internal medicine, pathogenesis, epidemiology, diagnosis, monitoring and treatment protocols. The journal is characterized by the rapid reporting of reviews, original research and clinical studies across all disease areas.

\section{Dovepress}

The manuscript management system is completely online and includes a very quick and fair peer-review system, which is all easy to use. Visit http://www.dovepress.com/testimonials.php to read real quotes from published authors. 